\title{
True Amplitude BiSAR Image Reconstruction via Backprojection and Image Domain Scaling
}

\author{
Can Evren Yarman ${ }^{a}$ and Birsen Yazıc ${ }^{b}$ \\ ${ }^{a}$ Houston Technology Center, WesternGeco-Schlum-berger, Houston, TX 77042 USA; \\ ${ }^{b}$ Electrical, Computer, and System Engineering Department, Rensselaer Polytechnic Institute, \\ Troy, NY 12180 USA;
}

\begin{abstract}
We consider a bistatic synthetic aperture radar (BiSAR) system operating in non-ideal imaging conditions with receive and transmit antennas traversing arbitrary flight trajectories over a non-flat topography; transmitting arbitrary waveforms along flight trajectories etc. $\mathrm{In}^{1}$ we developed a generalized filtered-backprojection (GFBP) method for BiSAR image formation applicable to such non-ideal imaging scenarios. The method puts edges not only at the right location and orientation, but also at the right strength resulting in true amplitude images. The main computational complexity of the GFBP method comes from the spatially dependent filtering step. In this work, we present an alternative, novel FBP method applicable to non-ideal imaging scenarios resulting in true amplitude images. The method involves ramp filtering in data domain and image domain scaling. Additionally, the method results in fast, computationally efficient implementation than that of GFBP methods.
\end{abstract}

\section{INTRODUCTION}

With the advent of uninhabited aerial vehicle technology and miniaturized sensor technology, it is envisioned that synthetic-aperture imaging technology will transition from using a single, dedicated platform to many small platforms operating in bistatic and multi-static mode. ${ }^{2}$ It is also expected that such SAR systems will operate in complex environments involving arbitrary flight trajectories, and employ diverse waveforms. Furthermore, scalability and survivability of such swarm of sensors will impose constraints on communication and computational resources. This means that image formation algorithms have to accommodate non-ideal imaging scenarios and be computationally efficient.

In, ${ }^{1}$ we developed a generalized filtered backprojection (GFBP) method for BiSAR image formation applicable to non-ideal imaging scenarios. The backprojection operator puts the visible edges at the right location and direction, but not at the right strength. When combined with the filtering, backprojection based imaging methods put the visible edges at the right location, with right direction and strength., ${ }^{1,}$ Thus, we refer to the resulting image as a true amplitude image.

$\mathrm{In}^{4}$ a fast filtered-backprojection (FBP) image formation method for monostatic SAR traversing linear flight trajectory was developed. This method, however, requires no slow-time and spatially-dependent filtering such as is required for GFBP methods developed for imaging in non-ideal monostatic and bistatic SAR scenarios. ${ }^{1,3}$ Moreover, the main computational complexity of these GFBP methods is due to the slow-time and spatiallydependent filtering step.

As an example, in, ${ }^{1}$ we showed that, assuming there are $\mathcal{O}(N)$ samples in both the fast-time and slow-time variables and that the image is of size $\mathcal{O}(N \times N)$, the computational complexity of the backprojection is of order $\mathcal{O}\left(N^{2} \log N\right)$ and the filtering is of order $\mathcal{O}\left(N^{3}\right)$. The question then is whether one can find a filter that would best approximate the strength of the edges of the image without comprising the computational complexity of the backprojection.

In this paper, we develop a novel, alternative approximate implementation of the GFBP method with $\mathcal{O}\left(N^{2} \log N\right)$ computational complexity. The method replaces computationally expensive data domain filtering with ramp filtering an image domain scaling. Our approach depends on repeated use of the forward and backprojection operators.

Further author information: (Send correspondence to Birsen Yazıcı)

Birsen Yazıcı: E-mail: yazici@ecse.rpi.edu

Can Evren Yarman: E-mail: yarman@ecse.rpi.edu

Airborne Intelligence, Surveillance, Reconnaissance (ISR) Systems and Applications VI, edited by Daniel J. Henry, Proc. of SPIE Vol. 7307, 73070E · C 2009 SPIE · CCC code: 0277-786X/09/\$18 · doi: 10.1117/12.818618 


\section{FORWARD MODELING AND GENERALIZED FILTERED-BACKPROJECTION}

Unless otherwise stated, the bold Roman, bold italic, and Roman small letters will denote points in $\mathbb{R}^{3}, \mathbb{R}^{2}$ and $\mathbb{R}$, respectively, i.e. $\mathbf{x}=\left(\boldsymbol{x}, x_{3}\right) \in \mathbb{R}^{3}$, with $\boldsymbol{x} \in \mathbb{R}^{2}$, and $x_{3} \in \mathbb{R}$.

Let $\gamma_{T}(s), \gamma_{R}(s) \in \mathbb{R}^{3}, s \in \mathbb{R}$, be the transmitter and receiver trajectories, respectively. We assume that the earth's surface is located at the position $\mathbf{x}=\left(x_{1}, x_{2}, \psi\left(x_{1}, x_{2}\right)\right)$, where $\psi: \mathbb{R}^{2} \rightarrow \mathbb{R}$, is a known smooth function, and scattering takes place in a thin region near the surface. Following, ${ }^{1}$ under the single scattering (Born) approximation, we model the received signal $d(s, t)$ as follows:

$$
d(s, t) \approx \mathcal{F}[V](s, t)=\int \mathrm{e}^{\left.-\mathrm{i} 2 \pi \omega\left(t-R_{T R}(s, \boldsymbol{x})\right] / c_{0}\right)} A_{T R}(\boldsymbol{x}, \omega, s) V(\boldsymbol{x}) d \omega d \boldsymbol{x},
$$

where $\boldsymbol{x}=\left(x_{1}, x_{2}\right), R_{T R}(s, \mathbf{x})=\left|\gamma_{T}(s)-\mathbf{x}\right|+\left|\mathbf{x}-\gamma_{R}(s)\right| / c_{0}$ is the total travel time, $c_{0}$ denotes the speed of light, $T(\boldsymbol{x})$ denotes the surface reflectivity, and $A_{T R}$ is a complex amplitude function that includes the transmitter and receiver antenna beam patterns, the transmitted waveform, geometrical spreading factors, etc. Here $t$ denotes the fast-time and $s$, which is referred to as the slow-time, parameterizes the trajectory. The complex amplitude term $A_{T R}$ can be approximated by $A_{T R}(\boldsymbol{x}, \omega, s) \approx J_{T R}(\omega, s)\left[4 \pi\left|\gamma_{T}(s)-\mathbf{x}\right|\left|\boldsymbol{\gamma}_{R}(s)-\mathbf{x}\right|\right]^{-1}$, where $J_{T R}$ is the product of transmitter and receiver antenna beam patterns at slow-time $s$.

Note that if we set $\gamma_{T}(s)=\gamma_{R}(s)$, for all $s \in \mathbb{R},(1)$ becomes a model for monostatic SAR imaging.

We assume that for some $m_{A}, A_{T R}$ satisfies the symbol estimate

$$
\sup _{(s, \boldsymbol{x}) \in K}\left|\partial_{\omega}^{\alpha} \partial_{s}^{\beta} \partial_{x_{1}}^{\rho_{1}} \partial_{x_{2}}^{\rho_{2}} A_{T R}(\boldsymbol{x}, \omega, s)\right| \leq C_{0}\left(1+\omega^{2}\right)^{\left(m_{A}-|\alpha|\right) / 2}
$$

where $K$ is any compact subset of $\mathbb{R} \times \mathbb{R}^{2}$, and the constant $C_{0}$ depends on $K, \alpha, \beta, \rho_{1}$, and $\rho_{2}$. This assumption is needed in order to make various stationary phase calculations hold; in fact this assumption makes the "forward" operator $\mathcal{F}$ a Fourier Integral Operator. ${ }^{5-7}$

The ideal image formation problem is to estimate $T$ from the knowledge of $d(s, t)$ for some range of $s$ and $t$. $\mathrm{In}^{1}$ the filtered-backprojection operator $\mathcal{K}$ was given as follows:

$$
\tilde{T}(\boldsymbol{z})=\mathcal{K}[d](\boldsymbol{z}):=\int \mathrm{e}^{\mathrm{i} 2 \pi \omega\left(t-R_{T R}(s, \mathbf{z}) / c_{0}\right)} Q_{T R}(\boldsymbol{z}, \omega, s) d(s, t) d \omega d s d t
$$

where

$$
\mathcal{B} d(\boldsymbol{z}):=\int \mathrm{e}^{\mathrm{i} 2 \pi \omega\left(t-R_{T R}(s, \boldsymbol{z}) / c_{0}\right)} d(s, t) d \omega d s d t .
$$

is the backprojection operator, $\mathbf{z}=(\boldsymbol{z}, \psi(\boldsymbol{z}))$ and

$$
Q_{T R}(\boldsymbol{z}, \omega, s)=\chi_{\Omega_{\boldsymbol{z}}}(\boldsymbol{\xi}(\omega, s)) \frac{\overline{A_{T R}(\boldsymbol{z}, \boldsymbol{\xi}(\omega, s))}}{\left|A_{T R}(\boldsymbol{z}, \boldsymbol{\xi}(\omega, s))\right|^{2}} \frac{1}{\eta(\boldsymbol{\xi}(\omega, s), \boldsymbol{z})},
$$

is the spatially-dependent data domain filter. In (5) $\chi_{\Omega_{z}}$ is a smooth cut-off function equal to one in the interior of $\Omega_{\boldsymbol{z}}=\left\{\boldsymbol{\xi}(\omega, s, \boldsymbol{z}) \mid A_{T R}(\boldsymbol{z}, s, \omega) \neq 0\right\}$ and zero in the exterior of $\Omega_{\boldsymbol{z}}$; and $\eta(\boldsymbol{\xi}(\omega, s), \boldsymbol{z})=|\partial(\omega, s) / \partial(\boldsymbol{\xi})|$ is the Jacobian (also known as Beylkin determinant) that comes from the following change of variables:

$$
(\omega, s) \rightarrow \boldsymbol{\xi}=\frac{\omega}{c_{0}} \nabla_{\boldsymbol{z}} R_{T R}(s, \boldsymbol{z}) .
$$

Let $\boldsymbol{\Xi}:=\nabla_{\boldsymbol{z}} R_{T R}(s, \boldsymbol{z}) / c_{0}$. Close examination of the Beylkin determinant shows that, it can be factored into two parts, a ramp filter and a $\boldsymbol{z}$ - and $s$-dependent filter:

$$
\frac{1}{\eta(\boldsymbol{z}, \boldsymbol{z}, \boldsymbol{\xi})}=|\omega|\left|\Xi_{1}(s, \boldsymbol{z}) \partial_{s} \Xi_{2}(s, \boldsymbol{z})-\Xi_{2}(s, \boldsymbol{z}) \partial_{s} \Xi_{1}(s, \boldsymbol{z})\right|
$$

where $\boldsymbol{\Xi}=\left[\Xi_{1}, \Xi_{2}\right]^{T}$ and $s$ and $\omega$ are understood to be $s(\boldsymbol{\xi})$ and $\omega(\boldsymbol{\xi})$. 


\section{TRUE AMPLITUDE IMAGING VIA SPATIAL SCALING}

We know that the backprojection operator puts the visible edges at the right location and right orientation in the backprojected image. Furthermore, ramp-filtered and backprojected image is approximately the true amplitude image up to an image domain scaling factor. To see this, we write

$$
\begin{aligned}
{[\mathcal{B}|\omega|][d](\boldsymbol{z}) \approx \mathcal{B}|\omega| \mathcal{F}[V](z) } & =\int \mathrm{e}^{\mathrm{i} 2 \pi \omega\left(R_{T R}(s, \boldsymbol{z})-R_{T R}(s, \boldsymbol{x})\right) / c_{0}} A_{T R}(\boldsymbol{x}, \omega, s)|\omega| V(\boldsymbol{x}) d \omega d \boldsymbol{x} d s \\
& \approx \int \mathrm{e}^{\mathrm{i} 2 \pi \boldsymbol{\xi} \cdot(\boldsymbol{x}-\boldsymbol{z})} A_{T R}(\boldsymbol{x}, \boldsymbol{\xi}) \frac{|\omega(\boldsymbol{\xi})|}{\eta(\boldsymbol{\xi}(\omega, s), \boldsymbol{z})} V(\boldsymbol{x}) d \omega d \boldsymbol{x} d s \\
& =\int \mathrm{e}^{\mathrm{i} 2 \pi \boldsymbol{\xi} \cdot(\boldsymbol{x}-\boldsymbol{z})} A_{T R, \boldsymbol{\Xi}}(\boldsymbol{z}, \boldsymbol{x}, \boldsymbol{\xi}) V(\boldsymbol{x}) d \boldsymbol{\xi} d \boldsymbol{x} \\
& \approx \hat{V}_{\Omega_{\boldsymbol{z}}}(\boldsymbol{z}) \sigma(\boldsymbol{z})
\end{aligned}
$$

where

$$
\begin{aligned}
A_{T R, \boldsymbol{\Xi}}(\boldsymbol{z}, \boldsymbol{x}, \boldsymbol{\xi}(\omega, s)) & =\frac{A_{T R}(\boldsymbol{x}, \omega, s)}{\left|\Xi_{1}(s, \boldsymbol{z}) \partial_{s} \Xi_{2}(s, \boldsymbol{z})-\Xi_{2}(s, \boldsymbol{z}) \partial_{s} \Xi_{1}(s, \boldsymbol{z})\right|}, \\
\hat{V}_{\Omega_{\boldsymbol{z}}}(\boldsymbol{z}) & =\int_{\Omega_{\boldsymbol{z}}} \mathrm{e}^{\mathrm{i} 2 \pi \boldsymbol{\xi} \cdot(\boldsymbol{x}-\boldsymbol{z})} V(\boldsymbol{x}) d \boldsymbol{\xi} d \boldsymbol{x}
\end{aligned}
$$

and

$$
\Omega_{\boldsymbol{z}}=\left\{\boldsymbol{\xi} \mid A_{T R}(\boldsymbol{x}, s(\boldsymbol{\xi}), \omega(\boldsymbol{\xi})) \neq 0\right\} .
$$

In other words, $\hat{V}_{\Omega_{z}}$ represents the best possible image we can form using the bi-static data collection manifold $\Omega_{z}$.

In (8) to (9), we use the linearization of the phase via the change of variables $(\omega, s) \rightarrow \boldsymbol{\xi}$; the assumption in (2) and stationary phase approximation. Here $\sigma(z)$ is the spatial scaling function that needs to be determined in order to perform the true amplitude imaging. Once $\sigma$ is determined, from (9), one can compute $V(\boldsymbol{z})$ from the ramp-filtered and backprojected data by

$$
\hat{V}_{\Omega_{z}}(\boldsymbol{z}) \approx \sigma^{-1}(\boldsymbol{z})[\mathcal{B}|\omega|][d](\boldsymbol{z}) .
$$

Next we determine the spatial scaling factor $\sigma^{-1}(z)$ that is required for the true amplitude imaging.

\subsection{Computation of the Spatial Scaling Factor $\sigma^{-1}$}

Let $d_{0}$ denote the original measured data. We first ramp filter and backproject the measured data and obtain $V_{1}(\boldsymbol{z})=[\mathcal{B}|\omega|][d](\boldsymbol{z})$. Let $d_{1}$ be the synthesized data using the image $V_{1}$ and the forward map $\mathcal{F}$, i.e., $d_{1}(t, s)=$ $\mathcal{F}\left[V_{1}\right](t, s)$. We use the synthesized data $d_{1}$ and form another image by ramp filtering and backprojection, i.e., $V_{2}(\boldsymbol{z})=[\mathcal{B}|\omega|]\left[d_{1}\right](\boldsymbol{z})$. Following the steps of $(9)$, we have

$$
V_{2}(\boldsymbol{z}) \approx V_{1}(\boldsymbol{z}) \sigma(\boldsymbol{z}) \approx \hat{V}_{\Omega_{\boldsymbol{z}}}(\boldsymbol{z}) \sigma^{2}(\boldsymbol{z}) .
$$

Thus we approximate the spatial scaling factor by

$$
\sigma^{-1}(\boldsymbol{z}) \approx V_{1}(\boldsymbol{z}) / V_{2}(\boldsymbol{z}),
$$

and form the approximate true amplitude image by

$$
\hat{V}_{\Omega_{z}}(\boldsymbol{z}) \approx V_{1}(\boldsymbol{z}) \sigma^{-1}(\boldsymbol{z}) .
$$



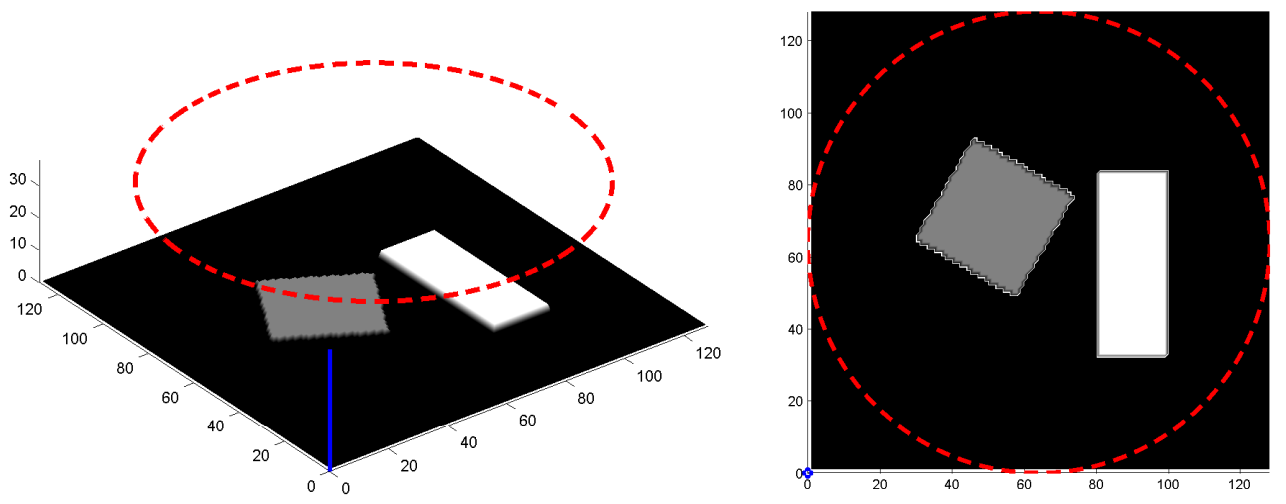

Figure 1. (Left) 3D and (right) 2D views of circular receiver flight trajectory (red dashed line) and the static transmitter (blue stright line). The axes labels are in kms.

\subsection{Computational Complexity of the True Amplitude Imaging via Image Domain Scaling}

Computational complexity of computing the scaling factor depends on the implementation of the forward projection operator. In our implementation, where we assumed a Dirac delta transmitted waveform, (see equation (17)), the computational complexity of the forward projection operator is of order $\mathcal{O}\left(N^{2}\right)$. Thus the computational complexity of computing the scaling function $\sigma^{-1}$ is dominated by the backprojection operator and is of order $\mathcal{O}\left(N^{2} \log N\right)$. Since the computational complexity of the scaling step is of order $\mathcal{O}\left(N^{2}\right)$, the total computational complexity of forming an approximate true amplitude image is dominated by the backprojection operator and is of order $\mathcal{O}\left(N^{2} \log N\right)$.

\section{NUMERICAL EXPERIMENTS}

In our numerical simulations, we considered a square target of size $5.5 \mathrm{~km}$ and a rectangular target of size $3.3 \mathrm{~km}$ by $8.8 \mathrm{~km}$ located in a scene of size $[0,22] \times[0,22] \mathrm{km}^{2}$ with their centers located at $(8.8,12) \mathrm{km}$ and $(15.4,10) \mathrm{km}$ (see Figure 1 ). We discretize the scene with $128 \times 128$ pixels, where $(0,0,0) \mathrm{km}$ and $(22,22,0) \mathrm{km}$ corresponding to the pixels $(1,1)$ and $(128,128)$, respectively (see Figure 2$)$.

We take $A_{T R}(\boldsymbol{x}, \omega, s)=1$, which corresponds to an isotropic transmit antenna radiating a delta-like impulse, an isotropic receive antenna and compensation of geometric spreading factors in the data. Then, using the definition of Dirac-delta function and its homogeneity property, we have

$$
d(s, t) \approx c_{0} \int \delta\left(c_{0} t-\left(\left|\gamma_{T}(s)-\mathbf{x}\right|+\left|\mathbf{x}-\gamma_{R}(s)\right|\right)\right) T(\boldsymbol{x}) d \boldsymbol{x} .
$$

We used a discrete version of (17) to generate our simulation data. The parameters we used correspond roughly to a system bandwidth of $.873 \mathrm{MHz}$. In these experiments, we considered a circular receiver flight trajectory $\gamma_{R}(s)=(11+22 \cos s, 11+22 \sin s, 6.5) \mathrm{km}$ uniformly sampled for $s \in[0,2 \pi]$ at 512 points, and a static transmitter located at $\gamma_{T}=(0,0,6.5) \mathrm{km}$ (see Figure 1 ).

We present the original $d_{0}$ and synthesized data $d_{1}$, reconstructed images $V_{1}, V_{2}$, the image domain scaling function and the approximate true amplitude image in Figure 3.

For comparison purposes, we also reconstructed an image using the GFBP method of ${ }^{1}$ (see Figure 4).

As can be see both the GFBP method of ${ }^{1}$ and the new ramp-filtered and backprojection and image domain scaling method produce qualitatively similar true amplitude images.

\section{CONCLUSION}

We presented a novel, computationally efficient true amplitude image reconstruction method for bistatic SAR image formation. In our future work, we will discuss extension of our approach to other synthetic aperture imaging modalities, such as synthetic aperture hitchhiker. ${ }^{8}$ 


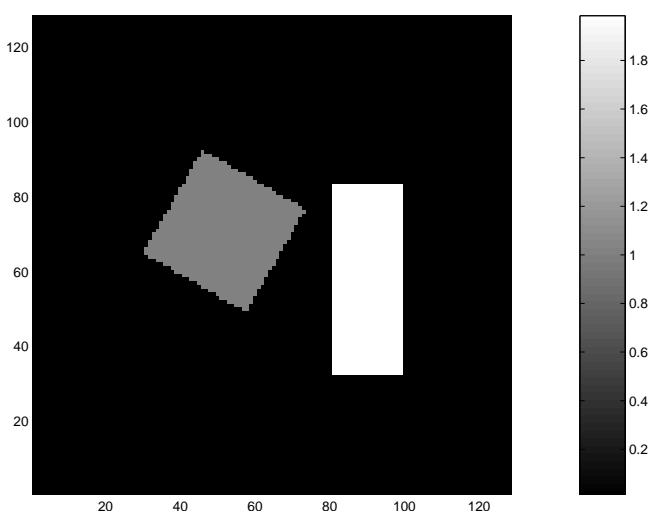

Figure 2. The scene used in numerical simulations. The axes are labeled according to the pixel number; this convention is also used for all the data and reconstructions below.

\section{Acknowledgment}

The authors would like to thank Dave Nichols and WesternGeco, Schlumberger. This work was partly supported by the Air Force Office of Scientific Research (AFOSR) under the agreement FA9550-07-1-0363 and by the National Science Foundation (NSF) under Grant No. CCF-08030672.

\section{REFERENCES}

[1] Yarman, C., Yazıcı, B., and Cheney, M., "Bistatic synthetic aperture radar imaging for arbitrary flight trajectories," IEEE Transactions on Image Processing 17, 84-93 (2008).

[2] Wicks, M., "Waveform diversity in intelligent sensor systems," in [IET Seminar Digests], www2.theiet.org/oncomms/pn/radar/WFP_WICKS01.pdf (2006).

[3] Nolan, C. and Cheney, M., "Synthetic aperture inversion for arbitrary flight paths and non-flat topography," IEEE Transactions on Image Processing 12, 1035-1043 (2003).

[4] Nilsson, S., Application of fast backprojection techniques for some inverse problems of integral geometry, $\mathrm{PhD}$ thesis, Linköping Studies in Science and Technology (1997). Dissertation No. 499.

[5] Duistermaat, J. J., [Fourier Integral Operators], Birkhauser, Boston (1996).

[6] Treves, F., [Introduction to Pseudodifferential and Fourier Integral Operators, volumes I and II], Plenum Press, New York (1980).

[7] Grigis, A. and Sjöstrand, J., [Microlocal Analysis for Differential Operators: An Introduction], London Mathematical Society Lecture Note Series, Vol. 196, Cambridge University Press, Cambridge (1994).

[8] Yarman, C. and Yazıcı, B., "Synthetic aperture hitchhiker imaging," IEEE Transactions on Image Processing 17, 2156-2173 (2008). 


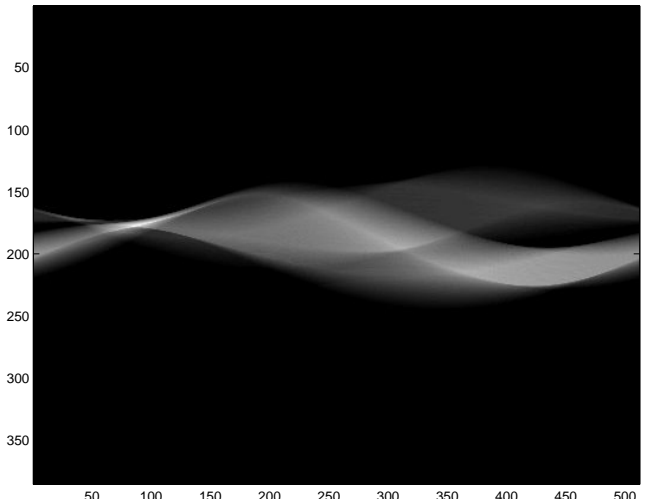

(a)

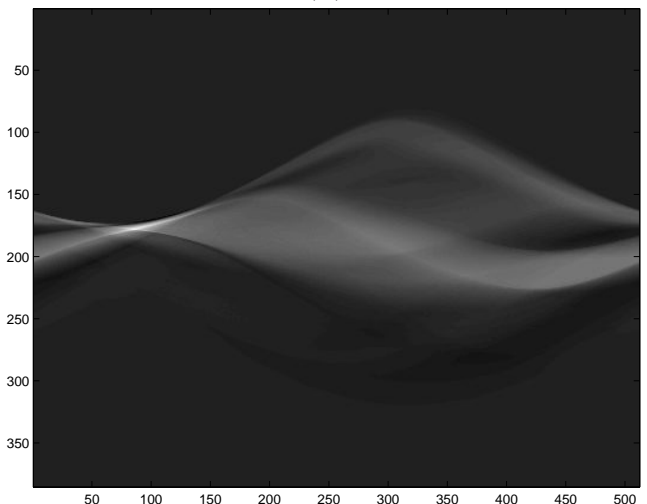

(c)

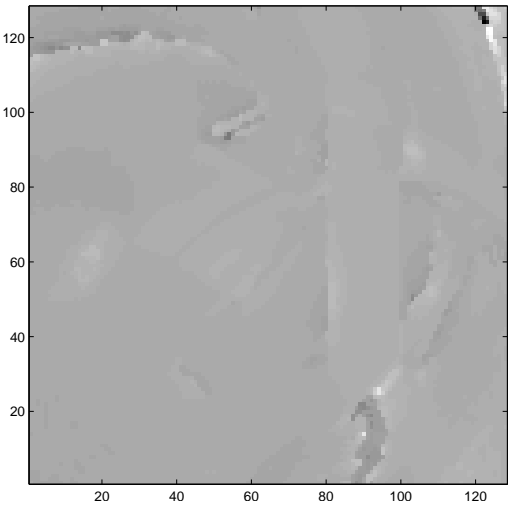

(e)

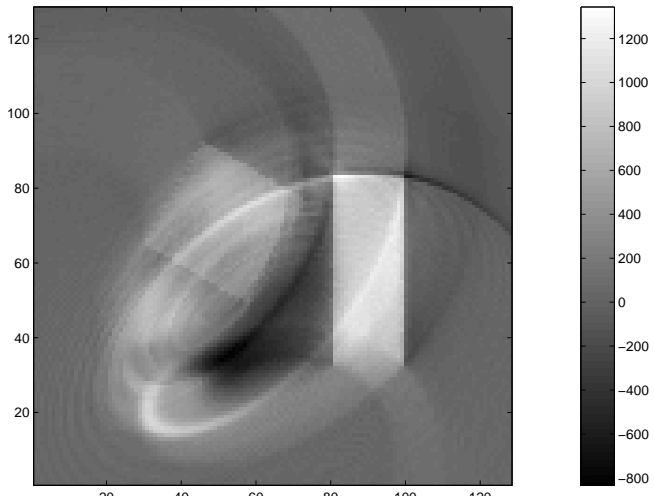

(b)

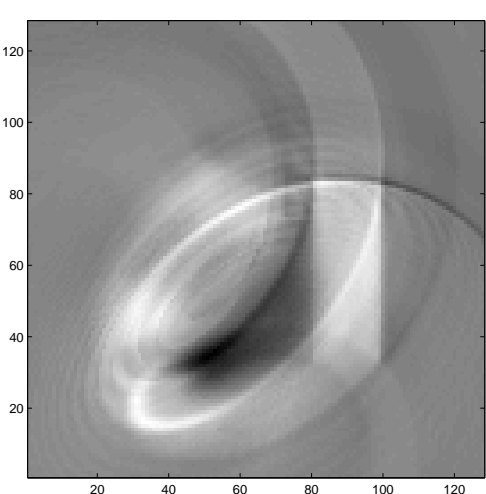

(d)

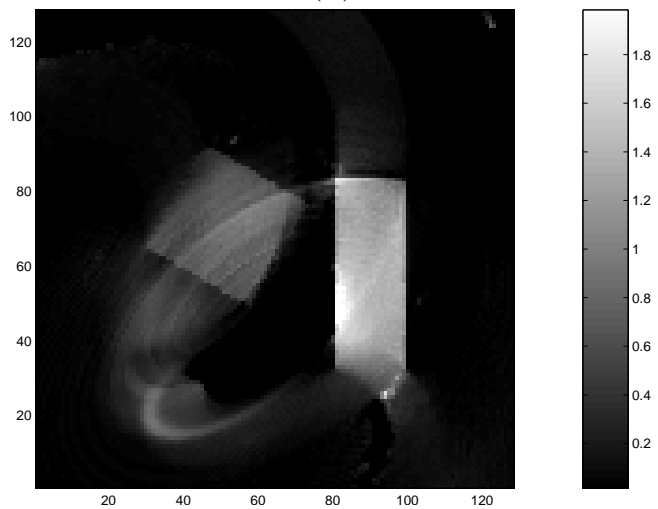

(f)

Figure 3. (a) Original data $d_{0}$ obtained from the scene $V$ shown in Figure 2. (b) Image $V_{1}$ obtained by ramp-filtering and backprojection of $d_{0}$. (c) Synthesized data $d_{1}$ from $V_{1}$. (d) Image $V_{2}$ obtained by ramp-filtering and backprojection of $d_{1}$. (e) The image domain scaling function $\sigma^{-1}$. (f) The approximate true amplitude image $\hat{V}_{\Omega^{z}}$. 


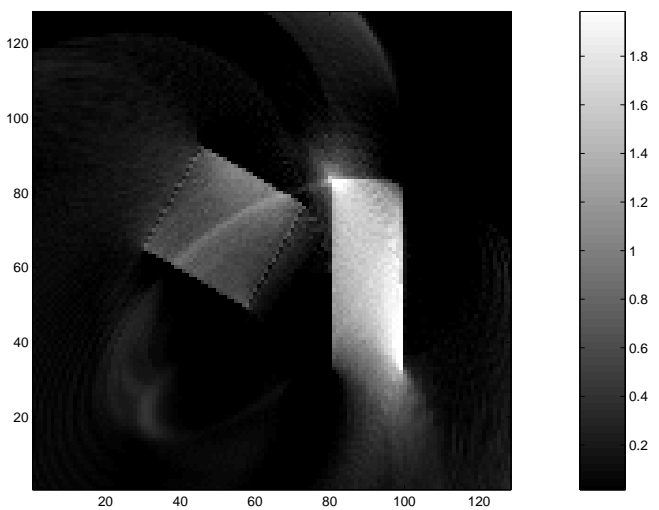

Figure 4. The bistatic SAR filtered-backprojection reconstruction ${ }^{1}$ of the original data $d_{0}$.

Proc. of SPIE Vol. 7307 73070E-7 